\title{
AFECCIÓN DE LAS AUTOVÍAS A SU ENTORNO RURAL: EXTREMADURA
}

\author{
Antonio Pérez Díaz \\ Departamento de Arte y Ciencias del Territorio. Universidad de Extremadura. Grupo de investigación GEDERUL \\ aperez@unex.es
}

Jorge J. Romo Berlana

Departamento de Construcción. Universidad de Extremadura. Grupo de investigación NEXUS jromober@unex.es

Manuel Sánchez Fernández

Grupo de investigación NEXUS manuel.1633@gmail.com

\section{RESUMEN}

La relación entre el transporte terrestre, y más específicamente el transporte por carretera a través de vías de gran capacidad o autovías, ha sido ampliamente estudiada desde la órbita urbana, por cuanto que éstas se estructuran como red de conexión de grandes núcleos a pesar de que discurren inevitablemente por territorio rural, como es el caso de la comunidad autónoma de Extremadura. Surge el interrogante de qué pasa con los pequeños núcleos ubicados al lado de estos corredores de transporte cuyo diseño se acometió a sus espaldas. Se estudia, por ello, cómo pueden ser afectados una serie de parámetros de territorios claramente rurales según su cercanía a una autovía. Los resultados indican distintos comportamientos de entornos rurales asociados a su grado de accesibilidad a una red de autovías.

Palabras clave: rural; autovía; grado de accesibilidad; población.

\section{ABSTRACT}

\section{Effects of highways on nearby rural areas: Extremadura}

The relationship between land transport, and more specifically road transport via high capacity roads or highways, has been extensively studied from the urban perspective, since such routes are structured as a network connecting large urban centres, although inevitably they run through rural territory, as is the case of the Autonomous Community of Extremadura. The question arises of what happens to rural villages located next to these transport corridors which were designed without taking them into consideration. A study has therefore been undertaken as to how a number of clearly rural parameters may be affected according to their proximity to a highway. The results indicate different behaviours of rural areas associated with their degree of accessibility to a network of highways.

Keywords: rural; highway; degree of accessibility; population.

\section{INTRODUCCIÓN}

La función vertebradora de las vías terrestres de comunicación (carretera y ferrocarril) ha ido ligada al desarrollo de los territorios, y más concretamente de los poblamientos conectados. Esta comunicación territorial se ha basado, hasta el desembarco de la revolución tecnológica de los modernos sistemas de 
comunicación, fundamentalmente en la calidad y eficacia de las seculares vías de transporte, que han condicionado las relaciones comerciales, económicas, sociales y culturales de los distintos territorios.

Las modernas vías de comunicación vienen marcadas por el uso generalizado del automóvil, cuya principal virtud es la autonomía y versatilidad del sistema: se llega desde cualquier parte a cualquier punto. De hecho, en los países desarrollados o medianamente desarrollados prácticamente todo el mundo tiene acceso al automóvil y, por tanto, al transporte por carretera. A lo largo de este proceso de generalización y masificación, la carretera impone su hegemonía frente al ferrocarril y pasa a ser el instrumento preponderante del sistema de transporte terrestre que, con mayor o menor acierto, vertebra el territorio. Los antiguos caminos ya no valen, es necesaria una reforma profunda de la red viaria con mayores exigencias técnicas que evolucionan según la demanda del nuevo mundo del motor.

El desarrollo de las grandes vías de comunicación surge ante la necesidad de unión de las grandes ciudades o principales núcleos urbanos de nuestra época, por ello, los pequeños núcleos o poblamientos son consideradas como manchas en el territorio por donde discurren esas grandes vías, que se concretan en largas cicatrices que seccionan el territorio de una forma más o menos agresiva, pero que, en todo caso, nacen de una función integradora encaminada a los grandes núcleos urbanos. Ya la red de calzadas romanas se diseñaba para unir las principales ciudades administrativas o núcleos de producción. Es suficiente echar un vistazo general a cualquier mapa carretero actualizado de España para comprobar que, salvo pocas excepciones vinculadas normalmente a la zona de influencia de las grandes urbes, las autovías se conforman según mallas que vertebran el país mediante la unión de sus principales ciudades, son corredores de ámbito interregional, nacional o continental. Se puede asegurar, por lo tanto, que estos corredores reflejan soluciones, con mayor o menor acierto, a un problema interurbano. La cuestión que planteamos es qué papel juega el mundo rural en esta dinámica.

Nuestra actual red viaria conjuga los grandes corredores carreteros (en España denominadas autovías) con la red secundaria (ya sean nacionales, autonómicas o provinciales) de forma que se llega prácticamente a todos lados. Ya no cabe hablar de zonas incomunicadas, pero sí de diferentes niveles o grados de comunicación.

Lo urbano se asocia a concentración, a densidad de actividades económicas, sociales, culturales, ambientales, etc. Todo dentro de un espacio geográfico relativamente reducido de naturaleza muy compleja por la cantidad de relaciones desarrolladas que, según los diferentes casos, ya sean geográficos, económicos, sociales, de comunicación o cualquier otra índole, estarán resueltos con mejor o peor resultado (Serratosa, 1993). Nadie duda de la complejidad de las relaciones que se desarrollan en un ambiente urbano o interurbano, que es foco de atención de numerosos estudios e investigaciones desde la aparición de la ciudad moderna. Por el contrario, lo rural se asocia a dispersión, extensión..., y cometeríamos craso error si concluyéramos estas cualidades como sinónimo de sencillez. De hecho, las relaciones humanas no dependen de dónde se ubiquen sino de su propia naturaleza, que se desarrolla igualmente en el entorno urbano y en el rural. Es decir, esas actividades económicas, sociales, culturales, ambientales, etc., son también fiel reflejo de la complejidad del mundo rural, y esta dispersión no las hace más sencillas, sino regidas por otros parámetros que tienen sus propias "leyes" de funcionamiento y que muchas veces dependen de una misma variable, pero con términos inversos u opuestos, p.ej., exceso de población versus escasez de población.

Así surge el interrogante: dado que las autovías están diseñadas por criterios de capacidad y accesibilidad de comunicación entre sistemas de ciudades ison inocuas a su entorno inmediato no urbano, por donde indefectiblemente discurren? Un examen detallado de los estudios existentes sobre las vías de comunicación y territorio abre un abanico de dimensiones tales que haría su análisis prácticamente inabordable, pero centrémonos en las vías de gran capacidad y su entorno rural. A pesar de ser un tema interesante existe relativamente poca bibliografía y trabajos de investigación específicos, que en general se acometen desde perspectivas sectoriales. "Se ha investigado poco sobre las rutas y el transporte en sí (trazados, estado ...) y menos aún sobre su relación con otros hechos sociales y económicos" (Madrazo Madrazo \& Frax Rosales, 2001, p. 31).

Desde el punto de vista de la planificación viaria encontramos trabajos de investigación sobre metodología de planes de desarrollo viarios pero a una escala territorial de gran extensión (incluso nacional o cuasi continental) en la que se estructura el territorio en un diagrama de nodos-itinerarios en los que el ámbito rural no se considera (López Suárez, 2007). Sin embargo, los planes de ordenación territorial en general se acometen a espaldas de los planes viarios, con las consecuencias que ello conlleva (Serratosa, 2002). 
Desde la perspectiva propiamente viaria, otros autores estudian la evolución del trazado de carreteras y su relación con el entorno, pero asociado al dúo vía-ciudad, tratando el ámbito rural en pocas ocasiones y términos difusos (Pozueta Echavarri, 2011); (Navarro Vera, 1988) que, en general, abordan la cuestión a una escala territorial al menos supracomarcal o son tratados teóricos de urbanismo que toman como referencia las ideas de ciudad lineal o parkway, entre otros. Son trabajos que consideran las vías como herramientas urbanizadoras de la ciudad y su entorno (Herce Vallejo, 1995), o analizan modelos urbanísticos de crecimiento de la ciudad, como la carretera-mercado en el que la propia vía ejerce de instrumento de conexión sobre el desarrollo generado en sus márgenes (Vecslir Peri, 2007).

Sí son numerosos los estudios históricos sobre la vinculación del camino y el territorio (Menéndez Pidal, 1951; Uriol Salcedo, 1992; García Arciniega, 2009) y encontramos interesantes trabajos arqueohistóricos de la relación entre las vías de comunicación y la ordenación territorial a nivel regional y comarcal de itinerarios principales (Nárdiz Ortiz, 1992).

Trabajos más recientes de investigación (Coronado Tordesillas, 2003) se centran más en la relación de tramos concretos de autovías y núcleos de población importantes existentes, es decir, entornos urbanos, con interesantes ideas en principio extrapolables al medio rural como el efecto barrera urbanístico de la autovía o enlaces como focos de generación de actividades. La evolución de las características de las vías y su apego o desapego al territorio con las consecuencias que ello trae, según las exigencias técnicas de las primeras, han sido también abordados (Nardíz Ortiz, 2002; Coronado Tordesillas \& Lázaro Rodríguez, 2008).

La exigencia de la sociedad de los últimos 30 años ha derivado a un compromiso de calidad ambiental anteriormente inexistente que se ha concretado en una normativa ambiental específica de obligado cumplimiento, que tiene como fin último preservar el medio natural en unas condiciones óptimas. (Hernández Fernández, 1995).

Desde la perspectiva del ámbito rural son incontables los estudios e investigaciones a escala global, nacional y regional que profundizan y analizan los comportamientos propios del mundo rural. En concreto, en el ámbito extremeño encontramos cómo desde el punto de vista social y económico (Gurría, Mora, Sánchez, \& Alberca, 1992) analizan la Vía de la Plata como eje vertebral del sistema de transporte bajo la perspectiva de relación vía-territorio, que es un precedente útil para desarrollar ideas y parámetros ligados a la demografía, actividad económica, accesibilidad, etc. El modelo territorial extremeño ha sido estudiado recientemente tanto desde la universidad (Campesino Fernández, 2003), como desde la propia Junta de Extremadura ("El Modelo Territorial de Futuro. Extremadura,"), con numerosos estudios desde la óptica de migraciones y pequeños municipios (Pérez Díaz, 2007; Pérez Díaz, 2006; Pérez Díaz \& Barrientos Alfageme, 2006), tanto de su estado actual como de su proyección de futuro y de la evolución del poblamiento en lo rural y urbano (Barrientos Alfageme, 2006).

Estos antecedentes parecen tender hacia una dicotomía entre lo técnico y lo social, si bien comparten el enfoque territorial, que puede llevar a una reflexión: parece claro que la complejidad urbana permite enfocar las distintas líneas de investigación bajo prismas claramente diferenciados. La idiosincrasia rural, sin embargo, empuja a un tratamiento más integrador. Las relaciones propias de lo rural se desarrollan en la actualidad en unas condiciones de equilibrio inestable; gran cantidad de parámetros que definen el modus vivendi rural, que presentan en nuestra reciente historia una línea generalizada de regresión (densidad de población, envejecimiento, diferente peso demográfico de la mujer, accesibilidad, renta económica, despoblación, servicios sociales, servicios de educación, actividad económica, calidad de naturaleza...) penden de condicionantes en general impuestos por la organización urbana de la sociedad actual, que pueden afectar de forma sensible a estos parámetros en desequilibrio.

El objetivo principal de la investigación es determinar si la presencia de una vía de gran capacidad de transporte por carretera afecta al comportamiento de estos parámetros en su entorno rural. La hipótesis de trabajo de partida es que la ubicación de los núcleos de población rural con respecto a una autovía puede afectar y, por lo tanto, condicionar el comportamiento, que a priori puede parecer ajeno, sobre dichos parámetros. Como objetivo secundario se pretende estimar, en su caso, cuáles son sus pautas de comportamiento en función de su cercanía o accesibilidad a una autovía.

No se considera en el presente estudio el análisis de todos los posibles parámetros, sería una tarea inabordable además de inconexa, por lo que se enfoca hacia aquéllos claramente representativos cuya disponibilidad de datos permitan obtener resultados suficientes para comprobar la consecución de los objetivos fijados y, en su caso, proponer otras líneas de investigación complementarias. Por todo ello se aborda el estudio de parámetros relacionados con la evolución poblacional (demográficos), con el comportamiento de la población (socio-demográficos) y con su actividad económica (socio-económicos). 


\section{METODOLOGÍA}

La investigación va a asociada a un enfoque integrador que relacione los dos pilares fundamentales del estudio planteado: autovía y entorno rural, por ello se deben analizar los factores más relevantes de ambos elementos. La obtención de resultados tangibles hace necesaria que la investigación vaya asociada a:

- Un marco geográfico concreto, suficientemente extenso, que tenga características indudables de ruralidad y esté dotado de una red de autovías moderna y completa.

- Un marco temporal que permita estudiar las evoluciones de los factores analizados.

- Disponibilidad de datos: no se trata de investigar nuevos datos, sino de usar en la investigación los ya existentes.

- Establecer un sistema de contraste que evite cometer errores sistemáticos.

La escala de trabajo va asociada a una doble componente territorial y temporal. Así, la unidad territorial elemental utilizada es el municipio, muy útil para el estudio del tamaño de asentamientos humanos y sus vinculaciones más inmediatas con su territorio inmediato, de la que se dispone de una serie de datos muy extensa (Zoido Naranjo \& Arroyo Pérez, 2003). Como unidad elemental temporal se adopta el año, que permite detectar las evoluciones de comportamiento de los distintos factores socio-demográfico-económicos analizados. Los datos utilizados para el desarrollo del trabajo referidos a población y sociedad se han obtenido de las bases de datos y publicaciones de distintos institutos de estadística oficiales: Instituto Nacional de Estadística [INE] y otros. Para la elaboración de mapas específicos durante la investigación se han utilizado diferentes capas del Instituto Geográfico Nacional [IGN].

Según la naturaleza del elemento estudiado se adoptan las herramientas técnicas adecuadas como Sistemas de Información Geográfica en entorno ARGIS para la elaboración de mapas (Arc Map) y análisis de redes arco-nodo (Network Analyst); tratamiento de datos numéricos mediante elaboración de matrices de doble entrada en entorno Excel y análisis de tendencias mediante gráficos de evolución bidimensional.

Gráfico 1. Esquema de proceso metodológico

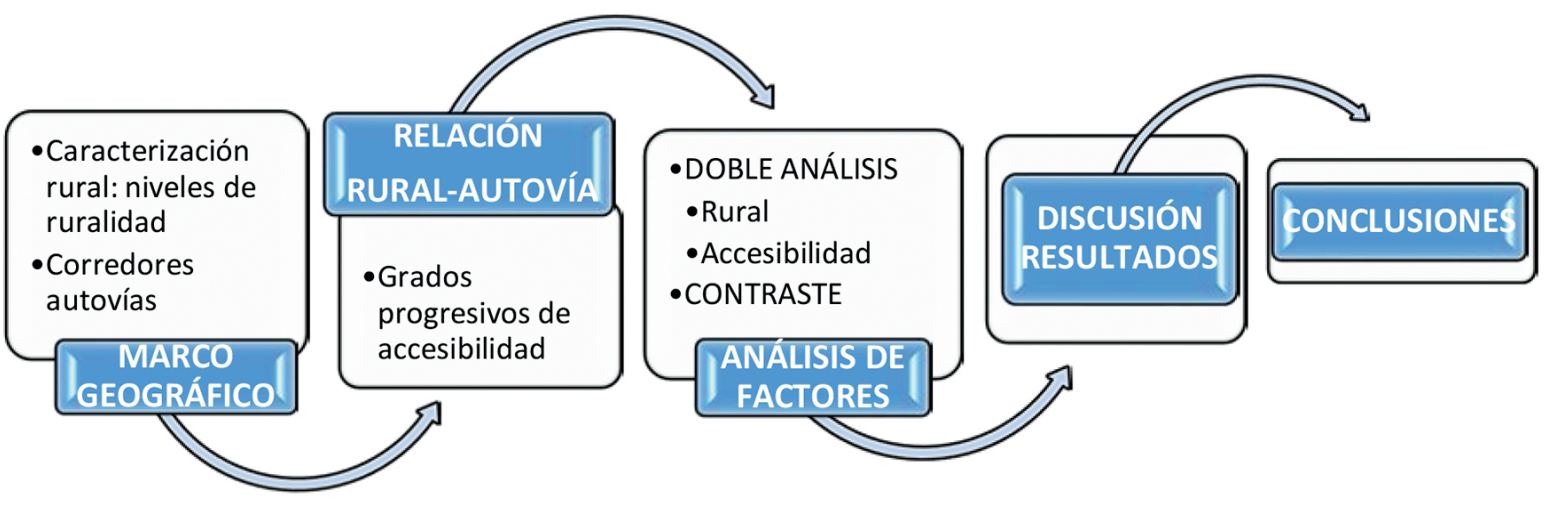

Elaboración propia.

El proceso metodológico (Gráfico 1), que parte del establecimiento de un marco geográfico adecuado, se basa, en primer lugar, en la caracterización de su ruralidad. La propia relatividad del término obliga a diferenciar, dentro de un territorio extenso claramente rural, entre distintos niveles de ruralidad; en este caso se han diferenciado hasta cuatro, de más rural a menos rural. A la vez que el análisis de ruralidad, se procede al análisis de la red de autovías con el estudio de los corredores por donde discurren.

La relación rural-autovía se fija, además de por los niveles de ruralidad, por la accesibilidad a las autovías, agrupando todos los municipios del ámbito estudiado según el nivel de conexión de su núcleo más representativo a la autovía más próxima, medido según el tiempo que tarda un vehículo por carretera en llegar hasta el enlace más próximo de autovía. Para ello se ha modelizado el sistema autovías-núcleos según una red topológica arco-nodo, fundamentada en la teoría de Grafos, composiciones de líneas, denominadas arcos y vértices denominados nodos (Garrido Palacios, 1995), junto con la red completa de carreteras extremeña. Los nodos de origen son los núcleos de los municipios, los nodos de paso las intersecciones de carreteras, los nodos de destino los enlaces de las carreteras con las autovías y los arcos son los tramos de carretera convencional existentes entre dos nodos. 
El grado de accesibilidad se interpreta como el tiempo utilizado por un vehículo tipo para recorrer la distancia desde el nodo origen (núcleo) al nodo destino (enlace más próximo de autovía). El cálculo se realiza asignando a cada tramo de carretera o arco una velocidad media representativa según su categoría (que depende del tráfico soportado, características propias de la vía y orografía de las zonas atravesadas) menor que su velocidad máxima de circulación, ya que se han contemplado travesías, intersecciones... Se ha considerado como vehículo tipo un turismo (realmente representativo para el concepto de accesibilidad). En el presente estudio se han establecido cinco niveles de accesibilidad de más cercano a más alejado.

Una vez establecida la caracterización territorial, según los cuatro niveles de ruralidad y los cinco grados de accesibilidad, se ha abordado el estudio exhaustivo de distintos parámetros de naturaleza claramente rural que nos permitan analizar y comparar su evolución en el tiempo con cadencia anual para, en su caso, poder establecer pautas diferentes o semejantes de comportamiento según los distintos niveles y grados fijados.

La detección de la posible relación del parámetro estudiado con la presencia de la autovía o su ruralidad se consigue con un doble análisis que considera, en primer lugar, como factor fijo el nivel de ruralidad y variable el grado de accesibilidad y, en segundo, de forma inversa, factor fijo la accesibilidad y variable la ruralidad (Gráfico 2). Este sistema permite objetivar el comportamiento de cada factor de forma independiente.

Gráfico 2. Esquema de doble análisis

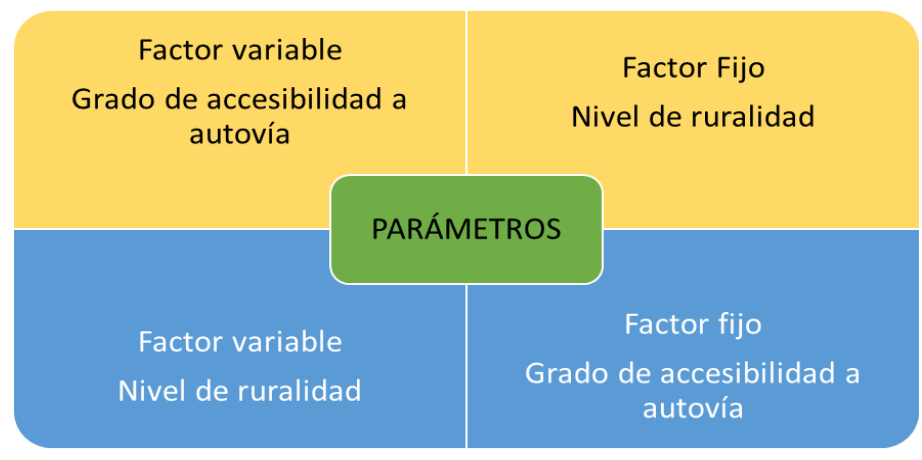

Elaboración propia.

Se han seleccionado parámetros de distinto espectro para un análisis global de la relación rural-autovía, agrupados en tres secciones diferenciadas que, a posteriori, permiten una interpretación conjunta de los resultados obtenidos: parámetros de carácter demográfico, socio-demográfico y socio-económico. Los datos empleados están disponibles en el INE y el Instituto de Estadística de Extremadura [IEEX]. Para garantizar la fiabilidad de la metodología adoptada se ha implementado un sistema de contraste que asegure que la conjugación autovía-entorno rural no esté contaminada por procesos externos no achacables a la relación entre estos dos pilares básicos. Por ello, se deben incluir en la investigación elementos en los que falte al menos uno de estos pilares, permitiendo la comparación de sus comportamientos frente a otros en los que sí se disponen de estos dos elementos básicos: autovía y rural. En un principio se consideró la selección de dos tipos de municipios de contraste, o patrones de referencia, que permitieran comparaciones de comportamiento: en primer lugar, municipios con bajo grado de accesibilidad $y$ alto nivel de ruralidad $y$, en segundo, alto grado de accesibilidad y bajo nivel de ruralidad. El inconveniente de la selección de estos municipios de contraste radica en que el patrón de referencia debe tener un grado de pureza tal que permita asegurar que su comportamiento no obedece a factores externos no considerados, lo que puede llevar al fracaso de la investigación. Para evitar este posible error sistemático, se ha implementado un sistema cuya virtud radica en que se analiza el comportamiento de todos los municipios del marco geográfico estudiado (Extremadura) independientemente de su nivel de ruralidad o grado de accesibilidad. El contraste queda garantizado con la comparación de los comportamientos de los distintos factores al variar progresivamente el carácter rural y/o la accesibilidad. Los cuatro niveles de ruralidad y cinco grados de accesibilidad justificados permiten una comparación y contraste de todo el ámbito estudiado que difuminan la posibilidad de sesgo sistemático respecto de un patrón de referencia. En la práctica supone establecer veinte escalones de comparación asociando los cuatro niveles de ruralidad con los cinco grados de accesibilidad, sistema que permite detectar con mayor precisión diferencias de comportamientos. 
Para cada parámetro se han elaborado tablas con los datos de todos los municipios de Extremadura por provincias, agrupados por su nivel rural y grado de accesibilidad. Se abarca todo el espectro de la población estudiada, no es una investigación estadística, sino de todo el poblamiento. De cada grupo y parámetro analizado se considera como valor representativo la media aritmética, que permite valorar tendencias comunes, evitando posibles sesgos por diferentes comportamientos de una cantidad de elementos no representativa. Es decir, cada parámetro, que puede tener naturaleza estadística según la fuente utilizada, es estudiado en toda su población considerando la media como valor representativo. El proceso ha requerido la elaboración de muchas, amplias y complejas tablas numéricas, a partir de las que se obtienen gráficos que simplifican el análisis de tendencias generales de comportamiento entre los distintos escenarios barajados. Además, la elección de una escala adecuada para cada factor también permite una correcta interpretación de las diferencias de magnitud.

\section{RESULTADOS}

Siguiendo la metodología definida se presentan los resultados obtenidos para los factores estudiados previo análisis y justificación de niveles de ruralidad y grados de accesibilidad.

\subsection{Niveles de ruralidad}

Extremadura cumple los condicionantes rurales característicos de población escasa poco participativa respecto el conjunto nacional y débil ocupación del territorio (Pérez Díaz, 2014). En términos comparativos con la población total española (según datos del INE de 01/01/2014) con 46.512.199 habitantes y una densidad media de $91^{\prime} 9 \mathrm{hab} / \mathrm{km} 2$, la población extremeña alcanza el 2'36 \% del total nacional ${ }^{1}$ ocupando el 8'2 \% de su extensión territorial. La densidad de población en Extremadura es tres veces y media menor. Distinguiendo entre provincias, para la misma fecha de referencia, se obtienen los siguientes datos (Tabla 1):

Tabla 1. Habitantes-densidades Extremadura por provincias

\begin{tabular}{|l|c|c|c|}
\hline & No Habitantes & Superficie $\left(\mathbf{k m}^{2}\right)$ & $\begin{array}{c}\text { Densidad población } \\
\left(\mathbf{h a b} / \mathrm{km}^{2}\right)\end{array}$ \\
\hline BADAJOZ & 690.929 & 21.766 & $31^{\prime} 7$ \\
\hline CÁCERES & 408.703 & 19.868 & $20^{\prime} 6$ \\
\hline EXTREMADURA & 1.099 .632 & 41.634 & $26^{\prime} 4$ \\
\hline
\end{tabular}

Fuente de los datos: IEEX. Elaboración propia.

En la actualidad la comunidad extremeña cuenta con un total de 388 municipios (165 en la provincia de Badajoz y 223 en Cáceres). Según el Registro de Entidades Locales del Ministerio de Hacienda y Administraciones Públicas el número total de municipios españoles es de 8.122, con una población media por municipio de 5.727 hab. El 2'36 \% de población se distribuyes en el 4'8 \% de municipios. La tipología de los municipios de cada una de las provincias según su extensión y población se indica para cada provincia en la Tabla 2. La comparación de los datos de Extremadura con los de España destacan por tener una densidad media de población 3'5 veces menor, proporción que aumenta a 4'5 en la provincia de Cáceres y disminuye a 2'9 en la de Badajoz.

Tabla 2. Caracterización de municipios por provincias, excluidos Badajoz y Cáceres

\begin{tabular}{|l|c|c|c|c|c|}
\hline \multicolumn{1}{|c|}{ PROVINCIA } & $\begin{array}{c}\mathbf{n}^{\mathbf{0}} \text { municipios } \\
(\mathbf{2 0 1 5 )}\end{array}$ & extensión $\left(\mathrm{km}^{2}\right)$ & $\begin{array}{c}\text { población } \\
\left(\mathbf{n}^{\mathbf{0}} \text { hab }\right)\end{array}$ & $\begin{array}{c}\text { extensión media } \\
\text { municipio }\left(\mathrm{km}^{2}\right)\end{array}$ & $\begin{array}{c}\text { población media } \\
\text { municipio (hab/munic) }\end{array}$ \\
\hline BADAJOZ & 164 & 20296 & 540412 & 124 & 3295 \\
\hline CÁCERES & 222 & 18118 & 312848 & 82 & 1409 \\
\hline
\end{tabular}

Fuente de datos: IEEX-Diputaciones-Otros. Elaboración propia.

1 El dato de población total de Extremadura el 01/01/2014 difiere ligeramente entre el INE y el IEEX, el primero determinal.096.421 y el segundo 1.099.632 hab. Esta diferencia del 0'29 \% no supone repercusión efectiva en el tema abordado. 
En cualquiera de los casos, densidad o población por municipio, Extremadura es claramente rural aplicando distintas clasificaciones de numerosos autores u organismos (Camarero Rioja, 1991; García Sanz, 1996; Serratosa, 2002; European Commisssion, Julio 1997; INE, 1981; LEY 45, 2007). A partir de ellas los municipios menores de 2.000 hab. se pueden considerar estrictamente rurales, entre $2.000 \mathrm{y}$ 5.000 hab. rural de medio tamaño y a partir de 10.000 hab. pueden categorizarse como urbanos.

Los porcentajes de la Tabla 3 denotan sin lugar a dudas la ruralidad de Extremadura desde la perspectiva poblacional, a la que suman aspectos también endémicamente rurales como pérdida de habitantes en poblaciones de menor tamaño, envejecimiento, alto índice de masculinidad o actividad agraria preponderante (Pérez Díaz, 2006), (Nieto Masot \& Gurría Gascón, 2008).

Tabla 3. Municipios de Extremadura \% por provincias y tamaño

\begin{tabular}{|l|c|c|c|c|}
\hline & \multicolumn{4}{|c|}{$\%$ de municipios según $\mathbf{n}^{\circ}$ de habitantes (2014) } \\
\hline \multicolumn{1}{|c|}{ PROVINCIA } & $<2000$ & $2000-5000$ & $5000-10000$ & $>10000$ \\
\hline BADAJOZ & $59 \%$ & $24 \%$ & $11 \%$ & $5 \%$ \\
\hline CÁCERES & $86 \%$ & $8 \%$ & $3 \%$ & $2 \%$ \\
\hline EXTREMADURA & $75 \%$ & $15 \%$ & $6 \%$ & $4 \%$ \\
\hline
\end{tabular}

Elaboración propia.

Según lo expuesto se han caracterizado todos los municipios extremeños según cuatro grados de ruralidad fijados por los límites de 2.000-5.000-10.000 hab. (Figura 1).

Figura 1. Municipios de Extremadura según población (2014).

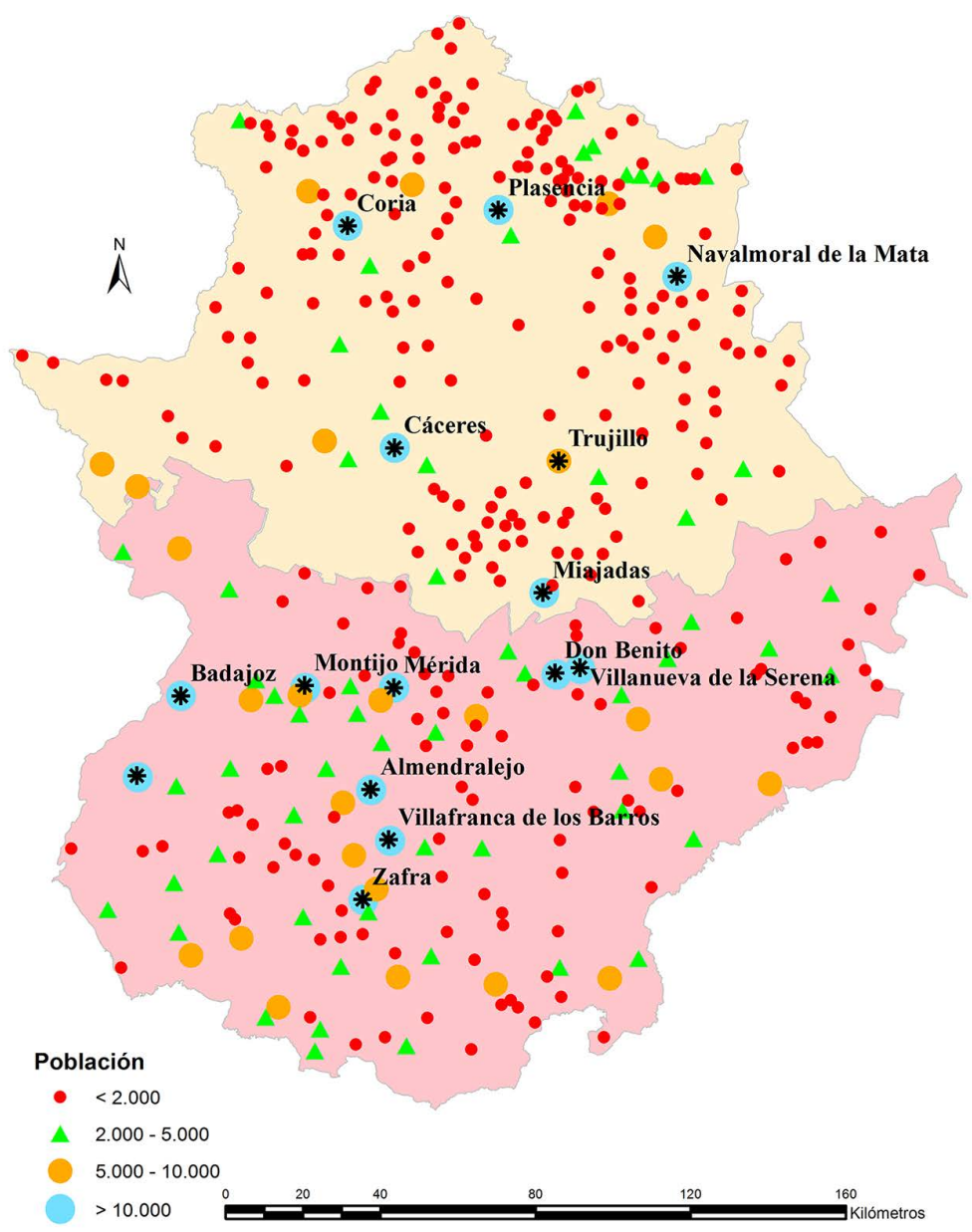

Elaboración propia. 


\subsection{Grados de accesibilidad a autovías}

La red de autovías de Extremadura es resultado de un largo proceso histórico, iniciado con las vías romanas, denostado en la edad media, recuperado en el siglo de la Ilustración, modernizado en el siglo XX y definitivamente consolidado en el inicio del siglo XXI, desembocando en la actualidad en unas autovías incardinadas en la red estatal y complementada con dos autovías autonómicas, todas concebidas y desarrolladas por planes sectoriales de transporte (Figura 2). Diversos estudios demuestran la coincidencia de gran parte de los corredores actuales con las calzadas romanas y los caminos del XVIXVIII. (Uriol Salcedo, 1985; Uriol Salcedo, 1977), extremo que se constata con la comparación de mapas en los que se ubican los caminos históricos y las autovías actuales.

Los principales corredores de autovías en la actualidad son (Figura 2):

- Corredor Noreste-Oeste: autovía A5 de Extremadura o del Suroeste. Las obras se terminan y ponen en servicio entre los años 1990 y 1993, salvo tramos puntuales poco representativos.

- Corredor Norte-Sur: autovía A-66 o vía de la Plata. Las fechas de puesta en servicio van desde 2001 a 2008.

- Corredores interiores: Corredor autonómico de la autovía EX-Al puesto en servicio entre 2005 y 2015. La también autovía autonómica EX-A2 no configura realmente un corredor sino un ramal de acceso a la A-5 inaugurado en 2006. A-58 entre Trujillo y Cáceres, puesto en servicio entre 2007.

La red extremeña de autovías vertebra la región por una línea media de norte a sur y otra de noreste a centro y oeste, complementadas por el eje transversal regional del tercio norte. A falta de los corredores del sureste (N-430 y N-432) y cierre de la EX-Al es una red bien estructurada con una sola conexión con Portugal y vacíos en los extremos este y oeste de la región.

Figura 2. Red de autovías en Extremadura en el contexto peninsular

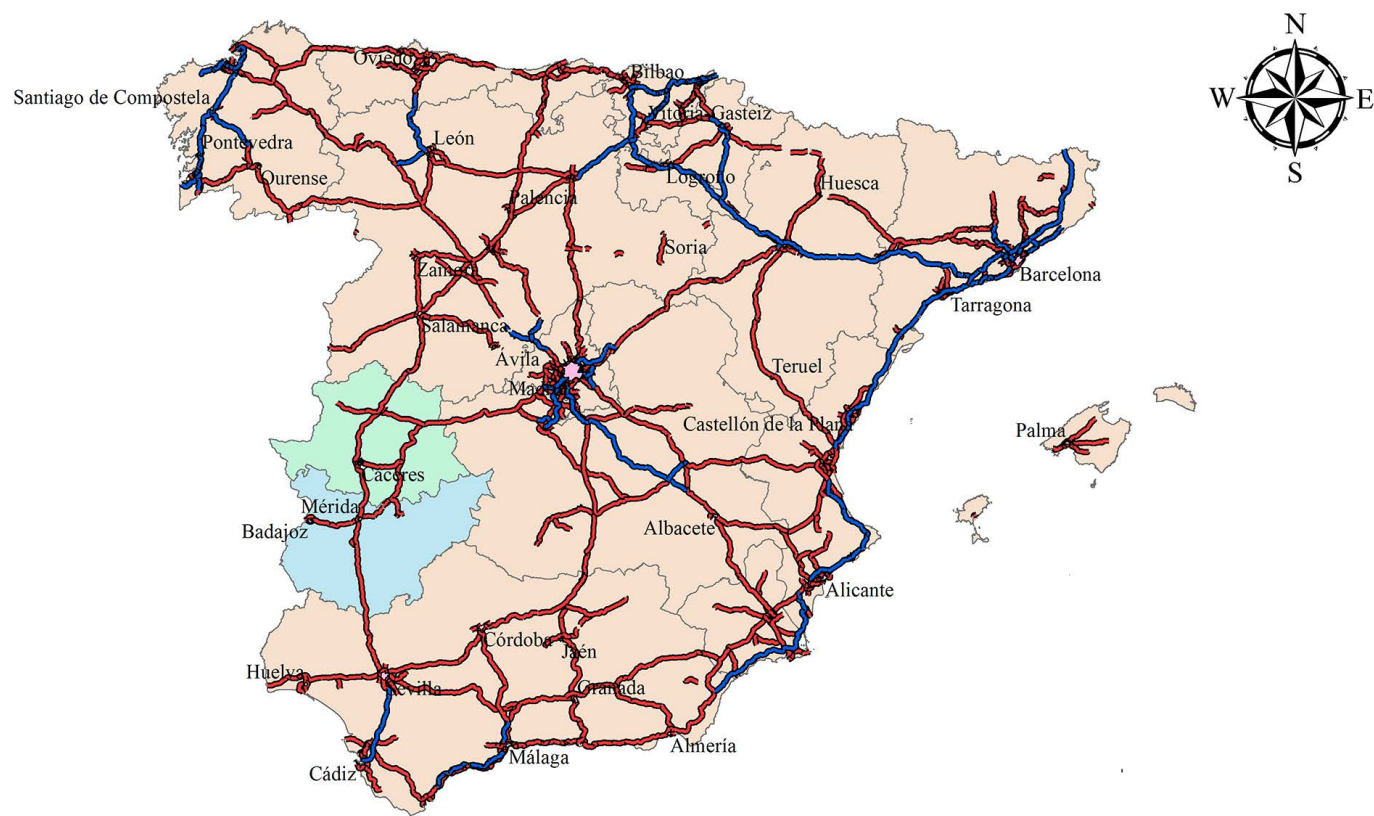

\section{Leyenda}

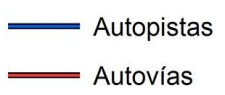

\section{1:9.000.000}

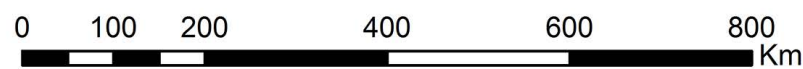

Elaboración propia.

Volcando los municipios según su nivel de ruralidad sobre el mapa de los principales corredores se puede estudiar su relación (Figura 3). Todas las poblaciones de más de 10.000 hab. están ubicadas al lado de una autovía. Sólo está aislada Olivenza en el oeste de Badajoz, Zafra se ubica a menos de $10 \mathrm{~km}$ conectada por una carretera nacional y Montijo conecta con la A5 por una carretera regional de longitud $5 \mathrm{~km}$. Se puede asegurar que se cumple la premisa de que los municipios menos rurales extremeños 
están conectados por autovía (a excepción de Olivenza que conecta con la A5 mediante una carretera autonómica de $25 \mathrm{~km}$ ). Por el contrario, sólo 6 de las 25 poblaciones entre 5.000 y 10.000 hab. se ubican junto la autovía. La dispersión por el total del territorio, alejadas de la autovía, es la nota dominante para municipios menores de 5.000 hab.

Según la metodología fijada la relación entre municipios y autovías se define por el grado de accesibilidad del núcleo más representativo del municipio a la autovía más próxima, medido según el tiempo que tarda un vehículo por carretera hasta el enlace más próximo de autovía. Las velocidades asignadas a cada vía se indican en la Tabla 4.

Tabla 4. Velocidades máxima y representativa del modelo de red

\begin{tabular}{c|cc}
\multicolumn{1}{c}{ TIPO DE CARRETERA } & $\begin{array}{c}\text { VELOCIDAD MÁXIMA } \\
(\mathrm{KM} / \mathrm{H})\end{array}$ & $\begin{array}{c}\text { VELOCIDAD } \\
\text { MEDIA (KM/H) }\end{array}$ \\
\hline CARRETERAS NACIONALES & 100 & 90 \\
\hline CARRETERAS AUTONÓMICAS & & 80 \\
\hline Básicas & 90 & 70 \\
\hline Intercomarcales & 80 & 50 \\
\hline Locales & 60 & 50 \\
\hline CARRETERAS PROVINCIALES & 60 & 40 \\
TRAVESÍAS REPRESENTATIVAS & 50 &
\end{tabular}

Figura 3. Mapa general de accesibilidad a municipios

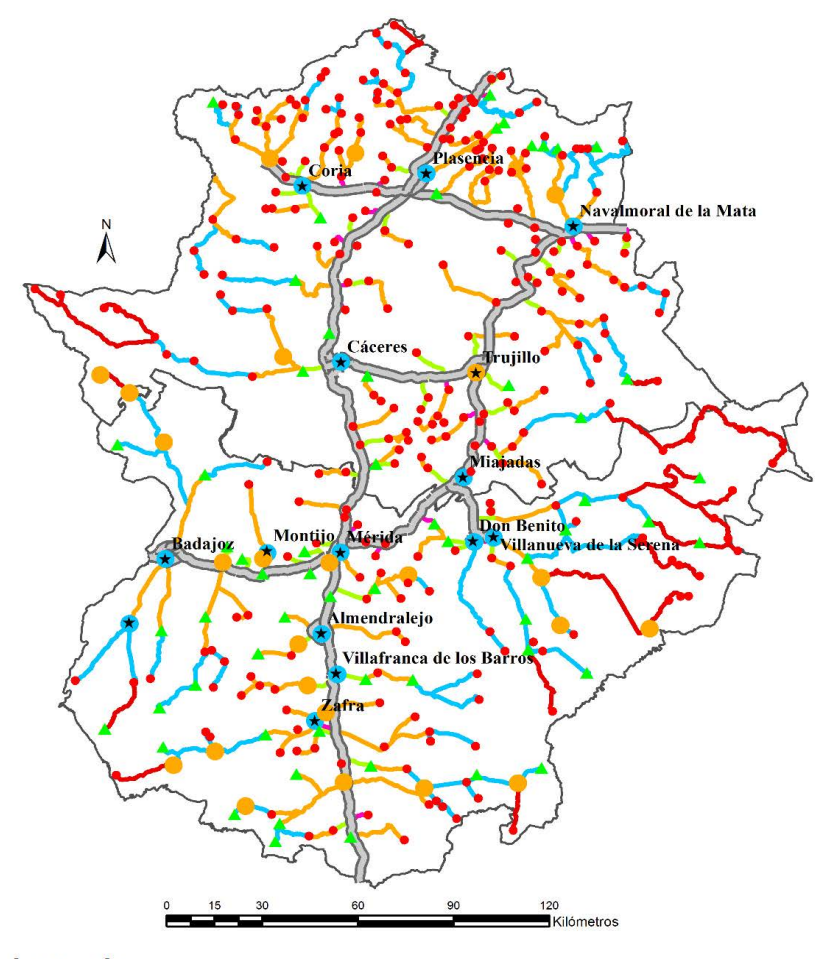

Leyenda

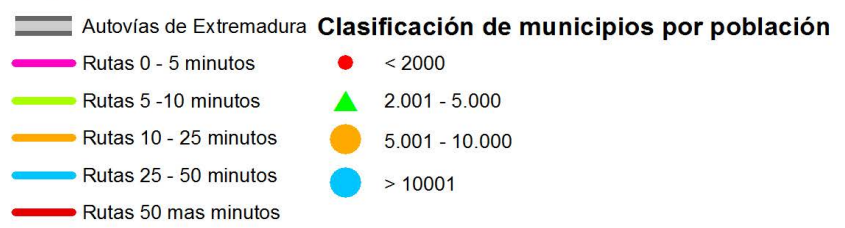

Elaboración propia. 
Para categorizar las accesibilidades de cada núcleo a la autovía, y evitar así una función continua difícil de interpretación, se establecen cinco niveles de proximidad o accesibilidad según los tiempos de recorrido empleados.

$\square$ Nivel temporal número 1: indica ubicación junto la autovía, la conexión con la autovía es prácticamente inmediata. Se fija un límite de 5 minutos.

$\square$ Nivel temporal número 2: indica cercanía a la autovía. El tiempo empleado en acceder a la misma es bajo. Se considera un intervalo de 5-10 min.

$\square$ Nivel temporal número 3: la autovía está a una distancia intermedia. El tiempo de acceso es apreciable. Se considera un intervalo de 10-25 min.

$\square$ Nivel temporal número 4: la autovía está alejada. El tiempo de acceso es elevado. Se considera un intervalo de 25-50 min.

$\square$ Nivel temporal número 5: la autovía está muy alejada. El tiempo de acceso es muy elevado. Se considera un umbral de $50 \mathrm{~min}$.

En la Figura 3 se representan las rutas óptimas de acceso a las autovías de todos los municipios, que se diferencian según códigos de colores asociados a los grados o niveles temporales de accesibilidad. Las ramificaciones demuestran la importancia en la estructura viaria de la A-66 de norte a sur, seguida del corredor de la A-5.

\subsection{Factores estudiados}

Con los veinte escalones de comparación o subgrupos establecidos se procede al análisis de la evolución de aquellos parámetros disponibles con una serie de datos suficientemente amplia que permita su estudio comparativo. Los parámetros seleccionados, cuyos datos se han extraído del INE y el IEEX, agrupados en tres secciones, son:

$\square$ Demográficos: evolución de la población.

๑ Índices socio-demográficos de: feminidad, vejez, sobreenvejecimiento, menores, dependencia, vegetativos (tasas de natalidad y mortalidad).

$\square$ Tasas socio-económicas de: paro (masculino y femenino), actividad por sectores (agraria, autónomos y general), vehículos a motor, líneas telefónica y entidades de crédito.

Para cada uno de los parámetros seleccionados se han realizado tablas de evolución con secuencia anual de las series disponibles y, a partir de ellas, se han elaborado gráficos según los escalones de comparación establecidos. La extensión de todas las tablas y gráficos trascienden al ámbito del presente artículo, por lo que a continuación se exponen algunos de los gráficos de evolución más representativos de algunos parámetros estudiados, que permiten analizar las diferencias de comportamiento para los distintos niveles de ruralidad y grados de accesibilidad respecto a la red de autovías.

Gráfico 3. Variación acumulada de población según corredor. Municipios $<2000$ hab. Provincia Badajoz

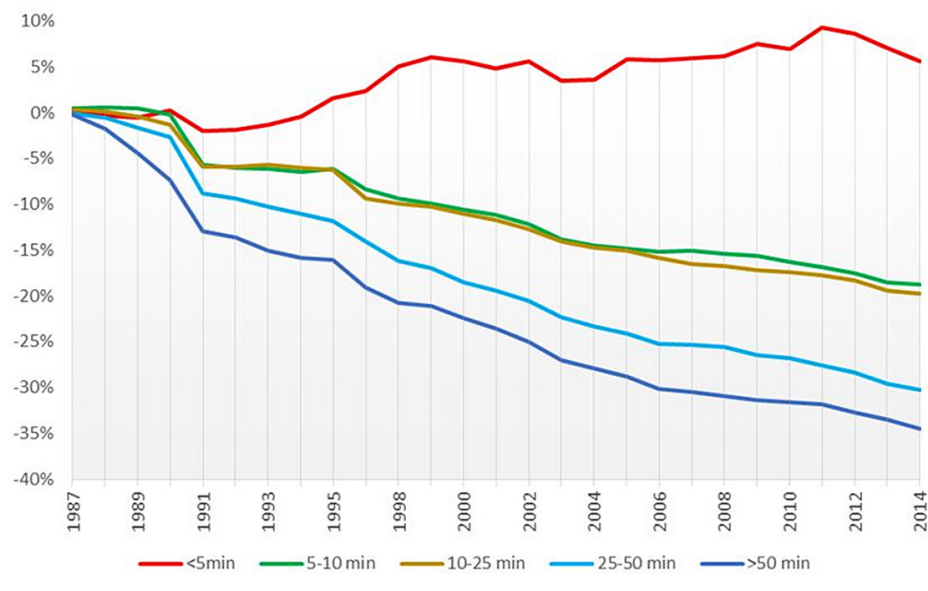

Elaboración propia. 
En los Gráficos 3 y 4 se refleja la evolución de la población para los municipios claramente rurales (<2000 hab). Las curvas de tendencia son claras en cuanto a cómo los municipios más cercanos a una autovía pierden menos población, incluso aumenta en la provincia de Badajoz. Por el contrario, las más alejadas presentan un alto gradiente de pérdida de población, gradiente que aumenta de manera progresiva según la distancia a la autovía. La jerarquía observada es clara en la provincia de Badajoz para los distintos grados de accesibilidad, que se reducen a dos en Cáceres: cercano y lejano.

Gráfico 4. Variación acumulada de población según corredor. Municipios <2000 hab. Provincia Cáceres

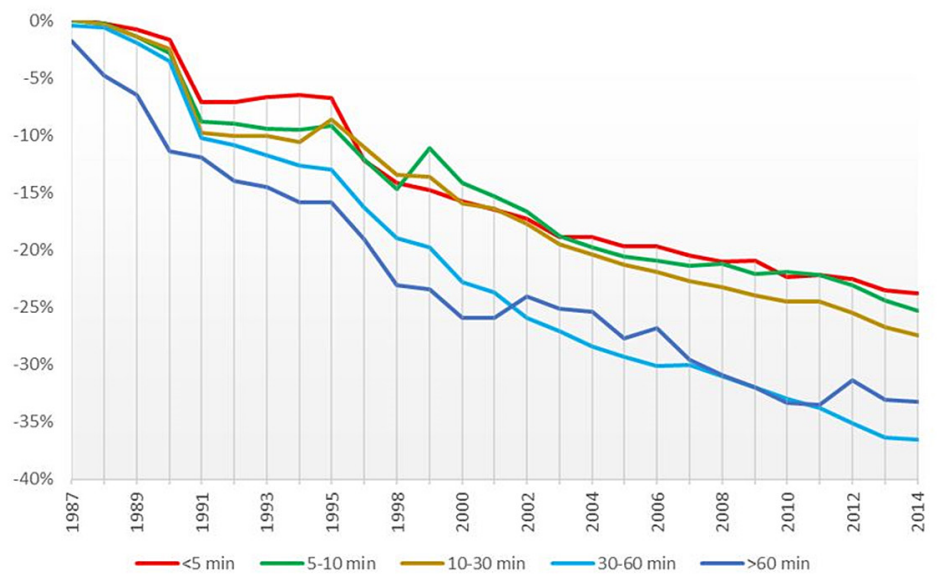

Elaboración propia.

En el Gráfico 5 se aborda la variación de población considerando fija la distancia a la autovía y variable la ruralidad según el tamaño. Se constata cómo, a igualdad de distancia, los municipios de la provincia de Badajoz presentan un gradiente de pérdida de población que disminuye según aumenta el tamaño, y cómo el comportamiento de las poblaciones intermedias (de 2000 a 10000 hab) es parejo.

Gráfico 5. Variación acumulada de población según tipo. Corredor 10-25 min. Provincia Badajoz

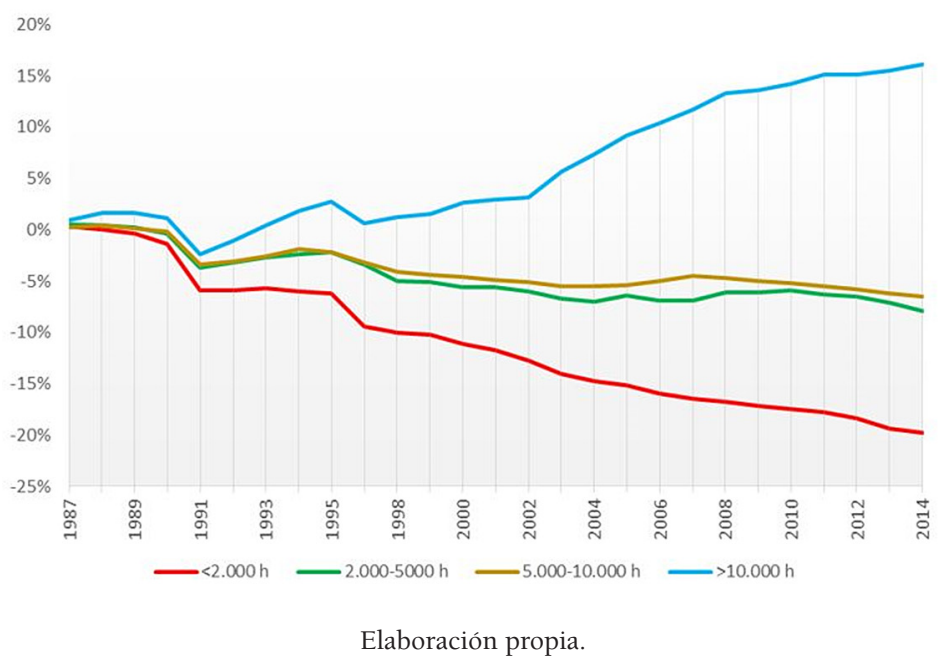

En el Gráfico 6, referido a la provincia de Cáceres, se repite el comportamiento visto en el gráfico anterior, con tasas de variación más diferenciadas, sin duda debido a la mayor ruralidad de los municipios cacereños. Sin embargo, los intermedios siguen con tendencias prácticamente iguales.

Si observamos las líneas de tendencia del Índice de Feminidad del Gráfico 7 (para la interpretación de este parámetro es conveniente el uso de líneas de tendencia que evite la distorsión de los dientes de sierra de los datos anuales) se observa que las poblaciones pequeñas más cercanas tienen un gradiente positivo frente el descenso de las lejanas y el comportamiento más neutro de las intermedias. Queda claro cómo las poblaciones pequeñas y cercanas recuperan tasa femenina mientras que las alejadas la pierden. 
Gráfico 6. Variación acumulada de población según tipo. Corredor < $5 \mathrm{~min}$. Provincia Cáceres.

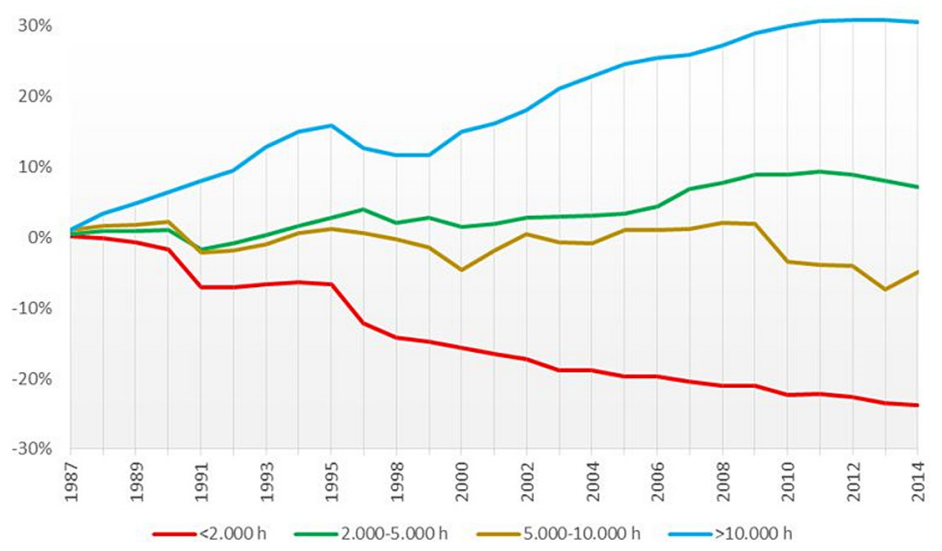

Elaboración propia.

Gráfico 7.Evolución índice de feminidad. Municipio <2000 hab. Provincia Badajoz

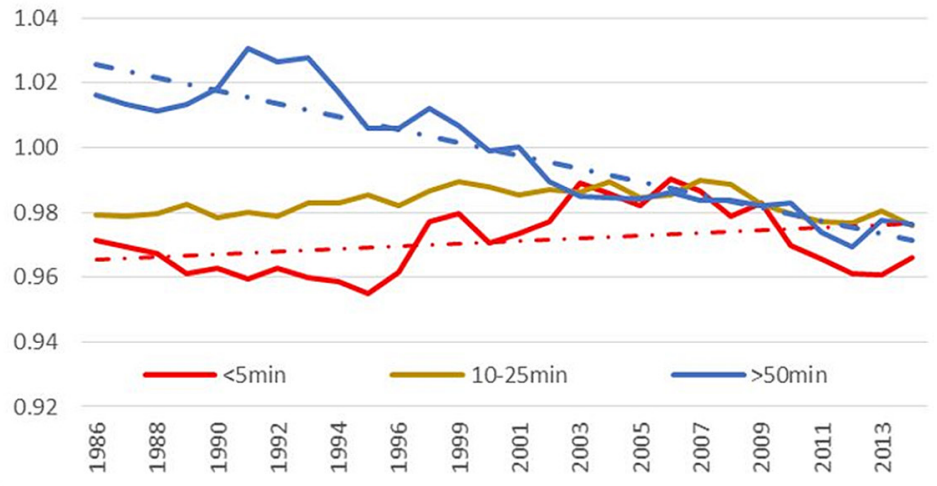

Elaboración propia.

Los valores del índice de dependencia de mayores (Gráfico 8) presentan una clara jerarquización para poblaciones pequeñas, según aumenta la distancia a la autovía mayor es el índice. Se vuelve a repetir la tendencia generalizada de que las poblaciones pequeñas cercanas a las autovías presentan valores menos regresivos, que las más alejadas, con clara diferencia de magnitud que aumenta con la distancia.

Gráfico 8. Evolución índice dependencia mayores según corredor. Municipios <2.000 hab. Provincia Badajoz

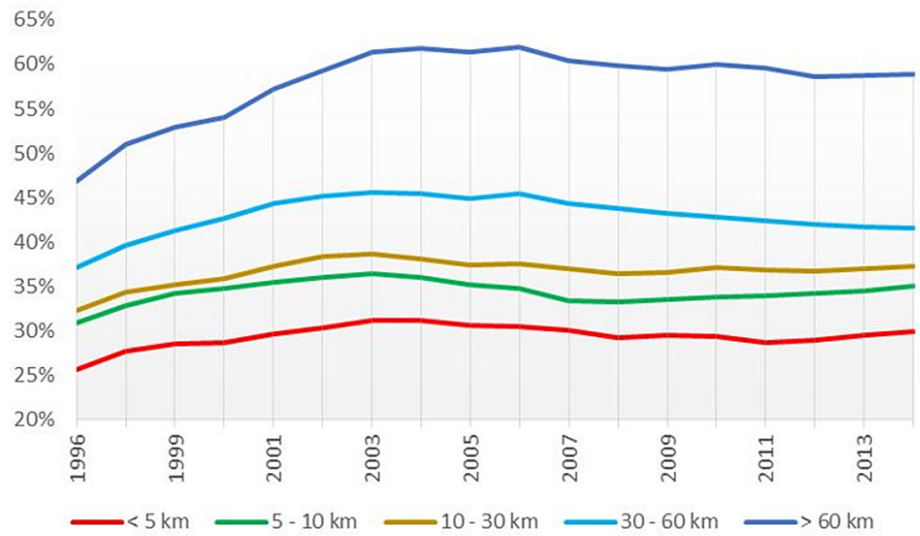

Elaboración propia. 
Para la interpretación de la tasa de natalidad es preciso utilizar de nuevo líneas de tendencia debido a la variabilidad anual del parámetro (Gráfico 9). La tendencia a la baja es general. Aun así, se observa un orden decreciente de magnitud de valores según aumenta la distancia a la autovía, de nuevo las poblaciones cercanas (en este caso de tamaño de 2000 a 5000 hab.) tienen mayores tasas de crecimiento.

Gráfico 9. Evolución tasa bruta natalidad según corredor. Municipios 2000-5000 hab. Provincia Cáceres

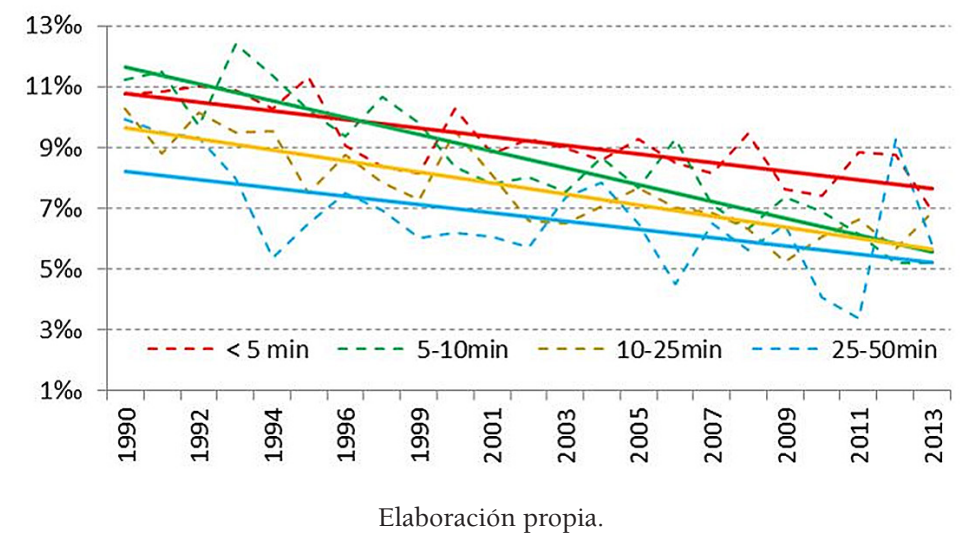

\section{DISCUSIÓN DE RESULTADOS}

Los resultados obtenidos reflejan que la presencia de una vía de gran capacidad de transporte por carretera sí afecta al comportamiento de ciertos parámetros en su entorno rural, detectándose distintas pautas según el nivel ruralidad y grado de accesibilidad a la red de autovías. Lógicamente, según se analice individualmente cada uno de los parámetros estudiados, los niveles de afección son distintos: encontrando en algunos casos comportamientos claramente diferenciados, líneas de tendencia o indiferencia en otros.

Una interpretación global de los resultados exige una visión integral del estudio realizado que filtre posibles fenómenos concretos no representativos del conjunto del ámbito geográfico estudiado. Para ello se ha elaborado una matriz resumen de interpretación de carácter cualitativo de todos los parámetros estudiados (Tabla 5). En ella se reflejan cuatro niveles de afección en el contexto autovía - entorno rural que permiten, con un sencillo análisis, establecer la intensidad de la relación detectada.

La relación más clara se encuentra en el comportamiento de la evolución de la población, seguida por parámetros que caracterizan a un entorno rural como el índice de feminidad o mayores, tasa de actividad agraria y vehículos industriales. Por el lado opuesto, se detecta que las tasas de ocupación laboral (masculina y femenina) no presentan variación de comportamiento, su ley es ajena a la hipótesis planteada. En un rango intermedio se encuadran el resto de parámetros sociodemográficos y socioeconómicos, en los que los comportamientos detectados presentan excepciones que no permiten establecer pautas generales, o bien, que la relación que parece clara en una provincia no lo es tanto en la otra.

De cualquiera de las formas, de los estudios y análisis abordados se colige que:

1. Una autovía fija población en su entorno rural más cercano, por cuanto que:

a. Los municipios más rurales y más accesibles pierden menos población.

b. Cuanto menos accesible es la autovía más población se pierde.

c. Los municipios de ruralidad media fijan más población cuanto mayor es el grado de accesibilidad a la autovía.

d. Los municipios no rurales tienen su dinámica propia independientemente de su relación de accesibilidad a la autovía.

2. La dinámica rural es distinta según la accesibilidad a una autovía por cuanto que:

a. Los parámetros socio-demográficos presentan en general un comportamiento más frágil y regresivo en los municipios más rurales y menos accesibles.

b. Los factores socio-económicos evidencian aún excesiva dependencia del sector agrario en los municipios más rurales y menos accesibles. 
Tabla 5. Matriz de interpretación de resultados

\begin{tabular}{|c|c|c|c|c|c|c|}
\hline & \multirow{2}{*}{\multicolumn{4}{|c|}{ NIVEL DE AFECCCIÓN }} \\
\hline & & & & & & \\
\hline CLASE & \multicolumn{2}{|c|}{ PARÁMETRO } & CLARO & APRECIABLE & $\begin{array}{c}\text { POCO } \\
\text { APRECIABLE }\end{array}$ & INAPRECIABLE \\
\hline DEMOGRÁFICO & \multicolumn{2}{|c|}{ Evolución Población } & $\mathrm{X}$ & & & \\
\hline \multirow{10}{*}{$\begin{array}{c}\text { SOCIO- } \\
\text { DEMOGRÁFICO }\end{array}$} & \multicolumn{2}{|c|}{ Índice Feminidad } & $x$ & & & \\
\hline & \multicolumn{2}{|c|}{ Índices Vejez } & & $x$ & $x$ & \\
\hline & \multirow{2}{*}{$\begin{array}{c}\text { Índices } \\
\text { Sobreenvejecimiento }\end{array}$} & Índice 4a Edad & & $x$ & & \\
\hline & & 4EEdad/3äEdad & & & & $x$ \\
\hline & \multirow{2}{*}{ Índices de Menores } & Índice Infancia & & $x$ & & \\
\hline & & Índice Juventud & & & $x$ & \\
\hline & \multirow{2}{*}{ Índices Dependencia } & ID Niños & & $x$ & & \\
\hline & & ID Mayores & $x$ & & & \\
\hline & \multirow{2}{*}{ Índices Vegetativos } & Tasa Natalidad & & & $x$ & \\
\hline & & Tasa Mortalidad & & & $x$ & \\
\hline \multirow{9}{*}{$\begin{array}{l}\text { SOCIO- } \\
\text { ECONÓMICO }\end{array}$} & \multirow{2}{*}{ Tasas de Paro } & Hombres & & & & $x$ \\
\hline & & Mujeres & & & & $x$ \\
\hline & \multirow{3}{*}{ Tasas de actividad } & Agraria & $x$ & & & \\
\hline & & Autónomos & & $x$ & & \\
\hline & & General & & & $x$ & \\
\hline & \multirow{2}{*}{ Tasas veh. Motor } & Automóviles & & & $x$ & \\
\hline & & Industriales & $x$ & & & \\
\hline & \multicolumn{2}{|c|}{ Tasa líneas tlfno } & & & $x$ & \\
\hline & \multicolumn{2}{|c|}{ Tasa entidades crédito } & & & $\mathrm{x}$ & \\
\hline
\end{tabular}

Elaboración propia.

\section{CONCLUSIONES}

De forma general se concluye que, según la hipótesis de trabajo planteada, los núcleos rurales presentan diferencias de comportamiento o evolución según su nivel de ruralidad y grados de accesibilidad a una autovía para ciertos parámetros. Esta relación es claramente apreciable en la evolución del número de habitantes y parámetros como índices de feminidad o mayores y tasas de actividad relacionadas con el sector primario. En cuanto a las tasas de paro no se observa influencia alguna, en este sentido las autovías se presentan inocuas, el problema del paro es un factor exógeno al ámbito de estudio. En un punto intermedio, de compleja concreción, encontramos el resto de factores estudiados como índices de dependencia, tasas vegetativas, así como factores asociados a actividad económica como tasas de autónomos o régimen general y vehículos motor.

Los resultados obtenidos son en general más patentes en la provincia de Badajoz, donde los factores analizados presentan líneas de comportamiento o tendencias más claras que en la provincia de Cáceres, con mayor número de excepciones. Causa de ello puede ser la diferente estructura de distribución poblacional de ambas provincias, menor dispersión poblacional y mayor número de habitantes medio en los municipios de Badajoz.

El trabajo desarrollado ofrece una nueva perspectiva a la relación de los sistemas de transporte modernos con su entorno, más o menos inmediato, no urbano. Se ha abordado con un enfoque ambicioso, con el estudio de numerosos factores asociados a distintos niveles de ruralidad y accesibilidad. En todo este espectro de factores y niveles se han detectado algunos con clara relación y otros sin ella, y una serie con grados intermedios que, sin duda, invita a proseguir la línea avanzada en esta investigación en otros 
ámbitos geográficos semejantes y diferenciados que permita la comparación de los resultados obtenidos. Concretamente, en el ámbito de estudio de Extremadura, parece lógico escudriñar otro nivel de ruralidad en la provincia de Cáceres que contemple el hecho de que, de los 189 municipios con menos de dos mil habitantes un total de 149 tienen menos de mil y 93 menos de quinientos. Otras líneas de investigación deben ir relacionadas con los sectores productivos diferenciadores de cada comarca que permitan comparar, p.ej., grandes zonas de regadío con otras de secano. Igualmente se pueden incluir en el análisis aspectos como las características físicas diferenciadoras del territorio.

De cualquier forma, la ya finalizada gran explosión de nuevas infraestructuras del transporte por carretera, abre una oportunidad para analizar la futura evolución de los factores estudiados, una vez que disponemos ya de una red consolidada que no prevé modificaciones ni ampliaciones relevantes y, por lo tanto, se puede seguir el estudio con uno de los pilares (la red de transporte), en la práctica y para el ámbito de estudio expuesto, constante. Este hecho, y el transcurrir del tiempo, difuminarán, tanto los posibles efectos cortoplacistas de inducción debidos a la aparición de nuevas infraestructuras, como los plazos de reacción del territorio ante esa nueva infraestructura y, permitirá analizar la relación rural-autovía con un sistema ya consolidado y normalizado en su funcionamiento.

\section{REFERENCIAS}

Barrientos Alfageme, G. (2006). La evolución del poblamiento en la dinámica rural-urbano. Norba. Revista de geografía, XI, 107-127. Recuperado de http://hdl.handle.net/10662/677

Camarero Rioja, L. A. (1991). Tendencias recientes y evolución de la población rural en España. Política y Sociedad, 8, 13-24. Recuperado de revistas.ucm.es/index.php/POSO/article/download/ POSO9191120013A/30546

Campesino Fernández, A. J. (2003). La ordenación territorial de la Extremadura del siglo XXI. Territoris, 4(4), 43-60. Rcuperado de http://ibdigital.uib.es/greenstone/collect/territorisVolums/import/ Territoris_2003v4.pdf\#page $=39$

Coronado Tordesillas, J. M. (2003). Tesis Doctoral: evolución de la relación entre carreteras y territorio: criterios territoriales de trazado. Los casos de estudio de los corredores Reinosa-Torrelavega (N-611) en Cantabria y Puerto Lápice-Santa Cruz de Mudela (N-IV) en Ciudad Real. Recuperado de https:// ruidera.uclm.es/xmlui/handle/10578/955

Coronado Tordesillas, J. M., \& Lázaro Rodríguez, F. J. (2008). Geometrías de las carreteras y del territorio. Ingeniería y territorio(84), 48-55. Recuperado de http://www.ciccp.es/revistalT/textos/pdf/06.\%20 Coronado\%20Tordesillas\%20y\%20Rodr\%C3\%ADguez\%20L\%C3\%Alzaro.pdf

El Modelo Territorial de Futuro. Extremadura. Taller de Ideas. Consejería de Fomento. JUNTA EXTREMADURA.

European Commisssion, D.-V. (Julio 1997). European Commission, Directorate General Agriculture (DG-VII).CAP 2000. Workimg Document. Rural Developments.July 1997.

García Arciniega, L. (2009). El saber encaminado: caminos y viajeros por tierras valencianas de la Edad Media y Moderna: Conselleria d'Infraestructures i Transport. Comunitat Valenciana.

García Sanz, B. (1996). Sociedad rural ante el siglo XXI: Ministerio de Agricultura, Pesca y Alimentación, Secretaría General Técnica. Recuperado de https://dialnet.unirioja.es/servlet/libro? codigo=69329

Garrido Palacios, J. (1995). La organización espacial de la red de carreteras en Aragón: aplicación metodológica de la teoría de grafos. Geographicalia(32), 83-102. Recuperado de https://dialnet. unirioja.es/servlet/articulo? codigo $=59842$

Gurría, J., Mora, J., Sánchez, R., \& Alberca, M. L. (1992). Vía de la Plata: eje vertebral en el sistema de transportes cacereño. Cáceres, Cámara Oficial de Comercio e Industria.

Herce Vallejo, M. (1995). Variante de la carretera y forma de ciudad. Tesis doctoral. Recuperado de http:// www.tdx.cat/handle/10803/6627

Hernández Fernández, S. (1995). Ecología para ingenieros. El impacto ambiental. Madrid: Colegio de Ingenieros de Caminos, Canales y Puertos.

Instituto de Estadística de Extremadura [IEEX]. Recuperado de http://estadistica.gobex.es/web/guest/ieex

Instituto Geográficon Nacional [IGN]. Recuperado de http://www.ign.es/ign/main/index.do

Instituto Nacional de Estadística [INE]. Recuperado de http://www.ine.es/

Instituto Nacional de Estadística [INE] (1981). Censo de Población, Tomo I. Vol. I,p. XXIII. 
Ley 45. (2007). Ley para el Desarrollo Sostenible del Medio Rural. BOE. Recuperado de https://www.boe. es/diario_boe/txt.php?id=BOE-A-2007-21493

López Suárez, E. (2007). Assessment of Transport Infrastructure Plans: a Strategic Approach integrating efficiency, cohesion and environmental aspects: E. López. Recuperado de http://oa.upm.es/373/

Madrazo Madrazo, S. M., \& Frax Rosales, E. (2001, p31). El transporte por carretera, siglo XVII-XX. Tst: Transportes, Servicios y telecomunicaciones(1), 31-53. Recuperado de https://dialnet.unirioja.es/ ejemplar/106311

Menéndez Pidal, G. (1951). Los caminos en la historia de España: Ediciones Cultura Hispánica.

Nardíz Ortíz, C. (2002). La transformación de un territorio de lugares en un territorio de redes. OP: Revista del Colegio de Ingenieros de Caminos, Canales y Puertos(60), 20-25. Recuperado de http:// europa.sim.ucm.es/compludoc/AA?articuloId=198022

Nárdiz Ortiz, C. (1992). El territorio y los caminos en Galicia: planos históricos de la red viaria. Colegio de Ingenieros de Caminos, Canales y Puertos de Galicia/Xunta de Galicia, Colección de Ciencias, Humanidades e Ingeniería.

Navarro Vera, J. R. (1988). La carretera y la ciudad: Travesías en el siglo XIX*'. Revista Obras Públicas. Unui 1988, 599-604.

Nieto Masot, A., \& Gurría Gascón, J. L. (2008). Las políticas rurales europeas y su impacto en Extremadura. Boletín de la Asociación de Geógrafos Españoles(48), 225-246.

Pérez Díaz, A. (2006). Los pequeños municipios ante los retos del desarrollo. Norba. Revista de geografía(11), 183-197. Recuperado de https://dialnet.unirioja.es/descarga/articulo/2280005.pdf

Pérez Díaz, A. (2007). La inmigración de retorno a Extremadura. Revista de estudios extremeños, 63(3), 1331-1364. Recuperado de https://papiro.unizar.es/ojs/index.php/geographicalia

Pérez Díaz, A. (2014). Treinta años de estancamiento poblacional y debilitamiento demográfico. Treinta años de Economía y Sociedad Extremeña. -1983-2013, 109-130.

Pérez Díaz, A., \& Barrientos Alfageme, G. (2006). Emigrantes retornados e inmigrantes: Extremadura ante el siglo XXI. Diputación de Badajoz, 147.

Pozueta Echavarri, J. (2011). Movilidad y planeamiento sostenible: hacia una consideración inteligente del transporte y la movilidad en el planeamiento y en el diseño urbano. Cuadernos de investigación urbanistica(30). Recuperado de http://polired.upm.es/index.php/ciur/article/view/244/240

Serratosa, A. (1993). Objetivos del planeamiento. Revista Obras Públicas n³327. Año 140, 7-12.

Serratosa, A. (2002). La escala comarcal y metropolitana en la ordenación del territorio. OP: Revista del Colegio de Ingenieros de Caminos, Canales y Puertos(60), 46-53. Recuperado de http://www.ciccp.es/ revistait/textos/pdf/6aserratosa60.pdf

Uriol Salcedo, J. I. (1985). Las calzadas romanas y los caminos del siglo XVI. Revista de obras publicas, 3237, 553-563. Recuperado de http://www.laviadelaplata.es/descargas/files/Caminos_del_Siglo_XVI.pdf

Uriol Salcedo, J. I. (1977). Apuntes para una historia del transporte en España. Los caminos de ruedas del siglo XVIII. Revista de obras publicas, 145-168. Recuperado de http://ropdigital.ciccp.es/pdf/ publico/1977/1977_marzo_3143_01.pdf

Uriol Salcedo, J. I. (1992). Hisoria de los Caminos de España. Siglos XIX y XX, VolII (Vol. 3242). Recuperado de http://polired.upm.es/index.php/urban/article/view/460

Vecslir Peri, L. (2007). Paisajes de la nueva centralidad. Urban(12), 34-55.

Zoido Naranjo, F, \& Arroyo Pérez, A. (2003). La población de España. Paper presented at the Tendencias demográficas durante el siglo XX en España. 